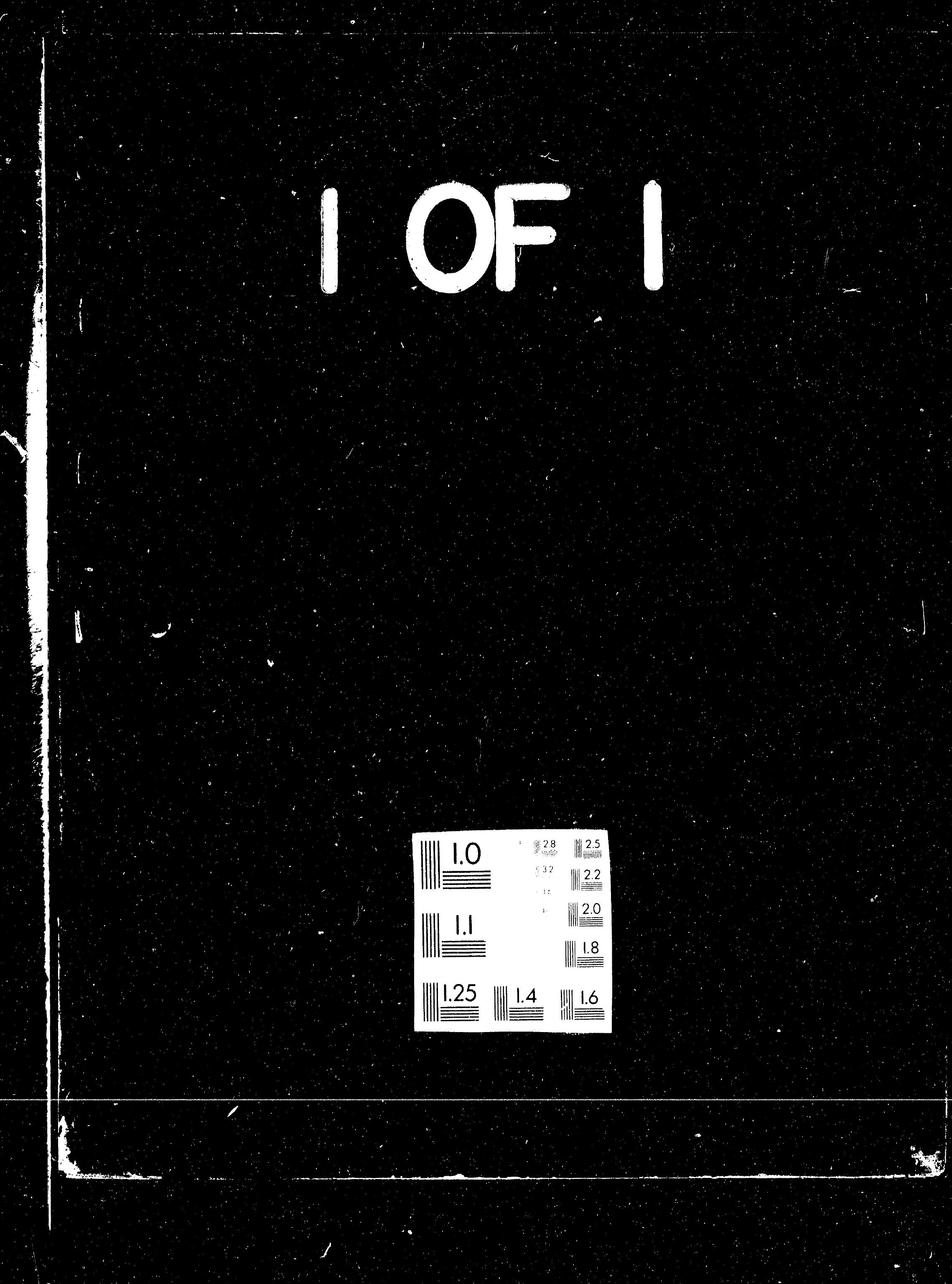




\section{(6. $921116-20$}

PNL-SA-21188

PNL-SA--21188

DE93 004841

ELECTRON-ELECTRON INTERACTIONS IN

FAST NEUTRAL-NEUTRAL COLLISIONS

R. D. DuBois

S. T. Manson

November 1992

Presented at the

12 th International Conference

on the Application of Accelerators

4

in Research and Industry

November 2-5, 1992

Denton, Texas

Prepared for

the U.S. Department of Energy

under Contract DE-ACU6-76RLO 1830

Pacific Northwest Laboratory Richland, Washington 99352

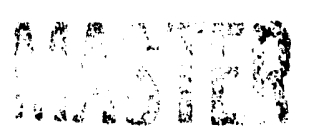




\title{
Electron-electron Interactions In Fast Neutral-neutral Collisions*
}

\author{
R.D. DuBois \\ Pacific Northwest Laboratory \\ Richland, WA 99352 \\ and \\ Steven T. Manson \\ Department of Physics and Astronomy \\ Georgia State University, Atlanta, GA 30303
}

Abstract:

Differential electron emission is studied for $50-500 \mathrm{keV} \mathrm{H}^{+}$and $\mathrm{H}$ atom impact on helium. Using the first Born formulation, it is shown that projectile electron-target electron interactions are expected to dominate the differential cross sections for low energy target electron emission induced by fast neutral projectile impact on any target. Measurements of the $15^{\circ}$ electron emission were made in order to investigate this prediction. For low impact energies, a constant ratio between the hydrogen atom and proton impact cross sections was found for emitted electron velocities less than half the projectile velocity, $v_{p}$. But as the collision energy increased, for electron velocities less than $0.25 V_{p}$, the cross section ratio increased as the emitted electron velocity decreased. This is interpreted as a signature of projectile electron-target electron interactions becoming dominant for distant collisions between neutral particles.

\section{DISCLAIMER}

This report was prepared as an account of work sponsored by an agency of the United States Government. Neither the United States Government nor any agency thereof, nor any of their employees, makes any warranty, express or implied, or assumes any legal liability or responsibility for the accuracy, completeness, or usefulness of any information, apparatus, product, or process disclosed, or represents that its use would not infringe privately owned rights. Reference herein to any specific commercial product, process, or service by trade name, trademark, manufacturer, or otherwise does not necessarily constitute or imply its endorsement, recommendation, or favoring by the United States Government or any agency thereof. The views and opinions of authors expressed herein do not necessarily state or icflect thuse of the United States Government or any agency therenf. 
Introduction:

As charged particles traverse matter, coulomb interactions between the nuclear charges and the electrons predominantly result in ionization processes. This means that in collisions involving clothed projectiles, meaning particles possessing bound electrons, projectile nucleus-target electron interactions lead to ionization of the target while target nucleusprojectile electron interactions lead to ionization of the projectile. However, in both cases, the nuclear charges are partially screened by bound electrons. Since the screening varies with the impact parameter of the collision, the target and projectile ionization are influenced to varying degrees depending on the momentum transferred to the ejected electron.

Generally, for fast collisions involving clothed particles, the dominant process is simple target (or projectile) ionization, i.e., ionization of one of the collision partners with the configuration of the other remaining unchanged. Since this process results from a first-order interaction between the electron that is ejected and the nucleus of the collision partner, which we abreviate as $e-n$, it is far more probable than the second-order $e-n$ process where an electron of particle $A$ interacts with the nucleus of particle $B$ and vice versa. This second-order process.leads to one of the collision partners being ionized and the other being excited.

However, first order interactions between target and projectile electrons can also lead to ionization-excitation of the collision partners. Since this multi-electron process results from a first-order interaction, it may not necessarily be small compared to the first order $e-n$ process-even at high energies. An example of where first-order projectile electron-target electron interactions, designated at e-e, are predicted ${ }^{1-3}$ to be important 
involves neutral-neutral collisions. In these collisions, e-e interactions are predicted to account for as much as $60-70 \%$ of the total target ionization cross section ${ }^{3}$ whereas for singly charged ion impact, they constitute only 10 $15 \%$ of the total target ionization cross section. The purpose of the current study is to explore the importance of projectile electron-target electron interactions in neutral-neutral collisions and to establish how they influence the differential electron emission.

\section{Theoretical Predictions:}

The first Born calculation can be used to illustrate the importance of first-order e-e interactions in fast clothed particle collisions. ${ }^{4.5}$ For simplicity, we shall limit our discussion to target ionization induced by fast particles with nuclear charge $\mathrm{Z}$ and $\mathrm{N}$ electrons in a single occupied electron subshell, e.g., H, He, $\mathrm{He}^{+}$projectiles. The doubly differential cross section, DUCS, for ionizing the target with the incident particle remaining in the ground state, i.e., the electron-nucleus process, is given by ${ }^{5}$

$$
\sigma(\epsilon)_{e-n}=\int A(\epsilon, K)\left[|Z-N F(K)|^{2}\right] d K .
$$

The DOCS for the projectile electron-target electron process, i.e., for ionizing the target and simultaneously exciting the incident particle, is given approximately by $^{5}$

$$
\sigma(\epsilon)_{e-e}=\int A(\epsilon, K)\left[N-N|F(K)|^{2}\right] d K .
$$

Note that all information about the target is contained in $A(\epsilon, K)$ while the term in brackets contains information about the screening of the incident particle's nuclear charge by its bound electrons. In these relations, $\epsilon$ is the energy of the ejected target electron, $\mathrm{kK}$ is the momentum transfer, and $F(K)$, the single electron form factor for the initial state of the projectile, $\mid i>$, is given by 


$$
F(K)=\left\langle|| e^{i k \cdot r} \mid i\right\rangle .
$$

By definition, $|F(K)|$ is bounded by 1 and 0 as $K \rightarrow 0$ and $\infty$, respectively. ${ }^{4-6}$ This corresponds to low and high electron emission energies, i.e., to large and small impact parameter collisions.

Note that the expressions for $\sigma(\epsilon)_{\mathrm{e}-\mathrm{n}}$ and $\sigma(\epsilon)_{\mathrm{e}-\mathrm{e}}$ are almost exactly the same, except for the terms in brackets, which we shall refer to as []$_{e-n}$ and []$_{e-e}$ respectively. The lower 1 imit of the integration, $k_{m i n}$, also differs for the two expressions, but, for the present discussion, this can be shown to make no major difference.

For neutral projectile impact, $\mathrm{Z}=\mathrm{N}$, and the bracketed term in Eq. becomes $\left[|N-N F(K)|^{2}\right]$ while the term in Eq. (2) is unchanged. Hence the first Born formulation predicts that as $F \rightarrow 1$, i.e., for $K \rightarrow 0$ which corresponds to low energy target electron emission, []$_{e-e} /[]_{e-n} \rightarrow \infty$. This means that e-e interactions will dominate the electron emission cross section for neutral projectiles colliding with any target. For larger emission energies, i.e., as $F \rightarrow 0$, the above formulation implies that []$_{e-e} /[]_{e-n} \rightarrow N^{-}$ ${ }^{1}$. However, since the e-e cross section, Eq. (2), depends rather critically upon the lower limit of integration, the ratio of cross sections, namely Eq. (2) divided by Eq. (1), may be somewhat different in the limit $F \rightarrow 0$. • Nevertheless, since low energy electron emission is the major contributor to the total target ionization cross section, e-e interactions also dominate the total ionization cross sections, as has been noted previously. ${ }^{2,3}$ Experimental Procedure:

In order to investigate the predicted importance of projectile electrontarget electron interactions in neutral-neutral collisions, doubly differential electron emission cross sections were measured for 50-500 keV 
hydrogen atom and proton impact on helium. Initially only laboratory electron emission angles of $15^{\circ}$ and $20^{\circ}$ have been investigated. The emphasis of this experiment is on the relative differences in the low energy electron emission for $\mathrm{H}$ and $\mathrm{H}^{+}$impact.

The experiment consisted of passing collimated, energy analyzed beams through a helium gas cell and collecting them in a Faraday cup. Electrons produced as the result of collisions within the cell exited through a slit and then were energy analyzed and counted using a parallel plate electron spectrometer and a channel electron multiplier. The system was calibrated using $300 \mathrm{keV}$ proton impact data normalized to absolute cross sections of ref. 7.

Ground state hydrogen atom beams were produced via electron capture by $\mathrm{H}^{+}$or dissociation of $\mathrm{H}_{2}^{+}$in a gas cell located prior to the target chamber followed by a strong electric field that removed the charged beam components and quenched any $H(2 s)$ metastable atoms. The $H$ beam "current" was monitored by biasing a portion of the beam Faraday cup positive and measuring the secondary electron current produced by impacting $\mathrm{H}$ atoms. In principle, absolute cross sections for $\mathrm{H}$ atom impact could be determined by converting the secondary electron yield to number of impacting $\mathrm{H}$ atoms. In practice, we chose to normalize the $\mathrm{H}$ atom and $\mathrm{H}^{+}$data in the binary encounter region where the cross sections are expected to be the same.

The detection efficiency for low energy electrons was improved by using a target cell consisting of an inner cylinder, biased at -2 volts, and a grounded, outer cylinder. High transmission grids covering the slits of both cylinders improved the field uniformity between the cylinders. Background signals from scattered electrons were reduced to less than $10 \%$ of the real 
signal in the $1 \mathrm{eV}$ energy range by coating all interior surfaces of the spectrometer with colloidal graphite and carbon soot, by shielding the spectrometer very we11, and by adding biased grids, just prior to the channel electron multiplier. With these improvements, the present data were found to reproduce within a factor of two in the $1 \mathrm{eV}$ range and to approximately $10 \%$ above $5 \mathrm{eV}$. However, relative uncertainties lexcept for those introduced in the normalization process) between the proton and hydrogen atom data tend to be much smaller than this, primarily because the data were collected in rapid succession using identical conditions.

Results :

In order to more closely observe differences in the differential electron emission spectra for $\mathrm{H}$ and $\mathrm{H}^{+}$impact, ratios of cross section were investigated. Specifically, it was assumed that the differential cross sections for $\mathrm{H}$ impact were given by a proton cross section multiplied by the square of an effective charge, $z_{\text {eff }}$, which depended on the emitted electron energy, $\epsilon$. Effective charges, determined in this fashion, are shown in Fig. 1 for a series of impact energies. Note that the abscissa is the electron velocity in units of the projectile velocity. Thus, electron loss from the projectile accounts for the peaked structure at $v=1$. Electron loss increases in importance relative to target ionization with increasing impact energies. Small impact parameter, binary collisions between the projectile nucleus and a target electron, are found in the region around $2 \cos \left(15^{\circ}\right)$. In this region the $\mathrm{H}$ atom and proton data were normalized to each other, thus $Z_{\text {eff }}=1$. Distant collisions result in low energy electron emission (the small $v$ region). It is in this region that electron screening effects become important and, hence, $Z_{\text {eff }}$ decreases. 


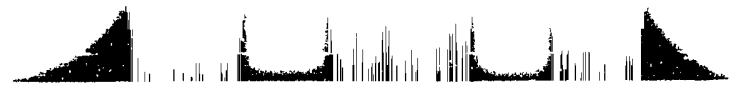

7

Fig. 1 shows that for low impact energies and for impact parameters leading to electron emission velocities less than $0.5 V_{p}$, the projectile is electron screens the nucleur charge, thus reducing the cross section by about a factor of 4 , i.e., $Z_{\text {eff }} \approx 0.45$, . However electrons with velocities exceeding that of the projectile are emitted in collisions where the impact parameter is sufficiently small that the $H$ ls electron cannot screen the nuclear charge, i.e., $Z_{\text {eff }}=1$. As the impact velocity increases, two major changes are noted. First, electron loss (projectile ionization) increases rapidly. This accounts for the growing peak centered at $v=1$. Second, $z_{\text {eff }}$ no longer remains constant for small $v$ but increases for $v<0.25$. This is attributed to projectile electron-target electron interactions becoming more important than projectile nucleus-target electron interactions, as was predicted. Note that at the higher impact energies shown, the electronelectron contributions are so important that target ionization resulting from neutral hydrogen impact exceeds that resulting from proton impact, i.e., Zeff $>1$, at least for forward angles of emission. A previous study ${ }^{8}$ using an $\mathrm{H}_{2} \mathrm{O}$ target implies this should also be found for large angles of emission. Conclusions :

By comparing differential electron emission cross sections resulting from hydrogen atom and proton impact on a helium target, it was shown that projectile electron-target electron interactions are major contributors to the target ionization for distant collisions. At high impact energies they were found to dominate projectile nucleus-target electron interactions for distant collisions. The present work only involved electron emission in the forward direction. Whether similar results are expected for all angles of emission must still be determined. 
Acknowledgments: This work was supported by the Office of Health and Environmental Research (OHER), U.S. Department of Energy, Contract No. DEAC06-76RLO 1830; by the National Science Foundation: and by U.S. Army Research Office Contract No. DAAL03-89-K-0098.

References:

1). K.L. Be11, V. Dose and A.E. Kingston, J Phys B $\underline{2}, 831$ (1969).

2). V.S. Nikolaev, V.S. Senashenko, V.A. Sidorovich, and V. Yu. Shafer, Zh Tekh Fiz 4ㅇ, 1399 (1978) and Sov Phys Tech Phys 23, 789 (1978).

3). G.H. Gillespie and M. Inokut, 1, Phys rev A 22, 2430 (1980).

4). J.S. Briggs and K. Taulbjerg, Topics in Current Physics 5, ed. I.A. Sellin, Springer-Verlag, 105 (1978).

5). R.D. DuBois and S.T. Manson, Phys Rev A $\underline{42}, 1222$ (1990).

6). J.H. McGuire, N. Stolterfoht and P.R. Simony, Phys Rev A 24, 97 (1981).

7). M.E. Rudd, L.H. Toburen, and N. Stolterfoht, Atomic Data and Nuclear Data Tables 18,413 (1976).

8). M.A. Bolorizadeh and M.E. Rudd, Phys Rev A 33,893 (1986). 
9

Figure Captions

Fig. 1. The effective nuclear charge (see text for details) of a hydrogen atom colliding with a helium target as a function of the velocity of the emitted electron, in units of the projectile velocity, $v_{p}$, and for various impact energies. The data are for a laboratory emission angle of $15^{\circ}$. 


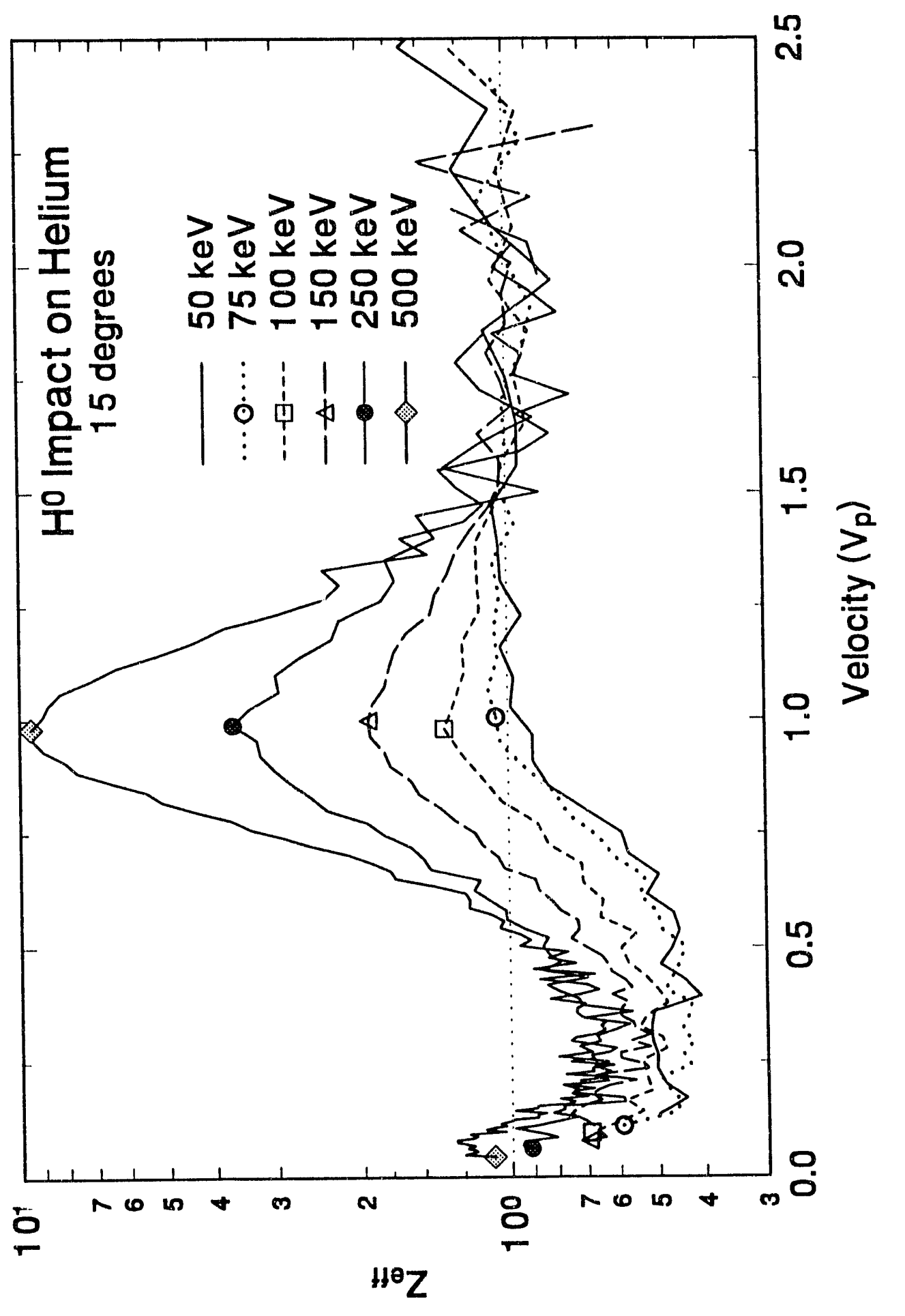



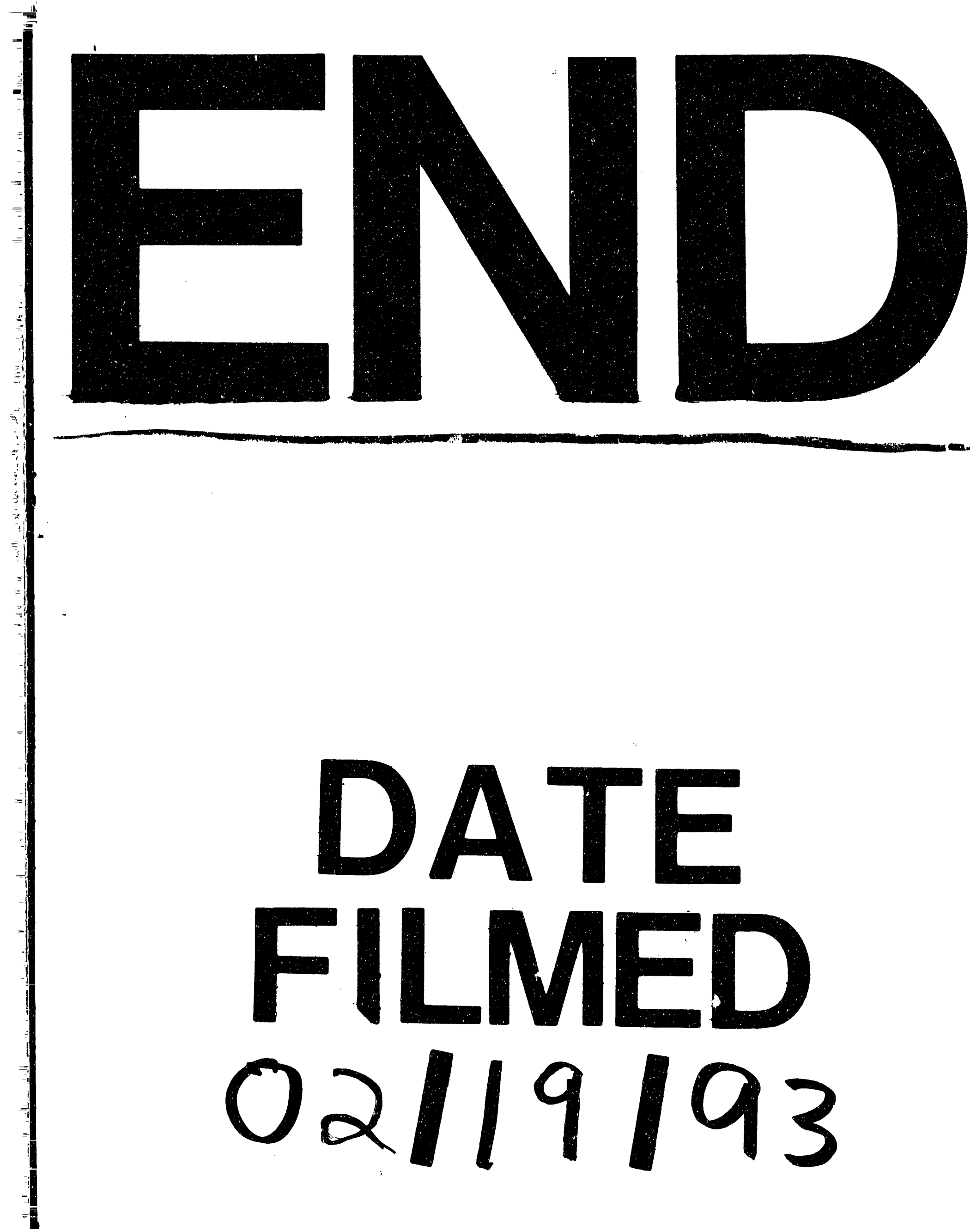
